\title{
An Application of High-Impedance Ground Planes to Phased Array Antennas
}

\author{
Romulo F. Jimenez Broas, Daniel F. Sievenpiper, Senior Member, IEEE, and Eli Yablonovitch, Fellow, IEEE
}

\begin{abstract}
The high-impedance surface was used as the antenna ground plane between two radiator elements to reduce the inter-element coupling between them. Measurement reveals the optimum performance occurred at the bandgap due to the suppression of surface currents in the ground plane.
\end{abstract}

Index Terms-Bandgap, coupling, phased array antennas, surface impedance, surface waves.

\section{INTRODUCTION}

$\mathbf{T}$ HE high-impedance ground plane is a metal sheet with a two-dimensional periodic resonant texture that suppresses surface waves in a desired frequency range called the bandgap [1]. In this bandgap, the resonance results in a suppression of surface waves by the high-impedance ground plane that can be beneficial in a variety of antenna applications [2], [3]. These include using the high-impedance ground plane to reduce the coupling between neighboring radiators sharing a common ground plane, as in a phased array [4].

For example, if two or more radiators share the same ground plane, they will suffer from coupling through two mechanisms: coupling through the surrounding space, and coupling through currents in the ground plane. To the extent that these surface currents can be minimized, the design of phased arrays can be simplified. The goal for using the high-impedance ground plane for arrays is that each antenna element in the array will radiate as if it is isolated, with less interference from neighboring elements. This will lead to the reduction of inter-element coupling.

The high-impedance ground plane used already proved to be useful in a cellphone handset geometry [5], [6]. This highimpedance ground plane was designed for $2.45 \mathrm{GHz}$, and it has physical dimensions as shown in Figs. 1-3. There is a parallel plate capacitance formed from the overlap between the two layers of metallic islands. An inductance is determined by the thickness of the board and its magnetic permeability. The overall result is an $\mathrm{LC}$ resonance centered at $2.45 \mathrm{GHz}$ with a bandgap from 2.35 to $2.55 \mathrm{GHz}$. It was this same high-impedance ground

Manuscript received April 30, 2004; revised August 17, 2004. The work described in this paper was performed when all of the authors were with the Department of Electrical Engineering, University of California at Los Angeles (UCLA).

R. F. J. Broas was with the Department of Electrical Engineering, University of California at Los Angeles, Los Angeles, CA 90095 USA. He is now with Raytheon, El Segundo, CA 90245 USA (e-mail: rbroas@yahoo.com).

D. F. Sievenpiper was with the Department of Electrical Engineering, University of California at Los Angeles, Los Angeles, CA 90095 USA. He is now is with HRL Laboratories LLC, Malibu, CA 90265 USA (e-mail: dfsievenpiper@hrl.com).

E. Yablonovitch is with Department of Electrical Engineering, University of California at Los Angeles, Los Angeles, CA 90095 USA.

Digital Object Identifier 10.1109/TAP.2005.844408

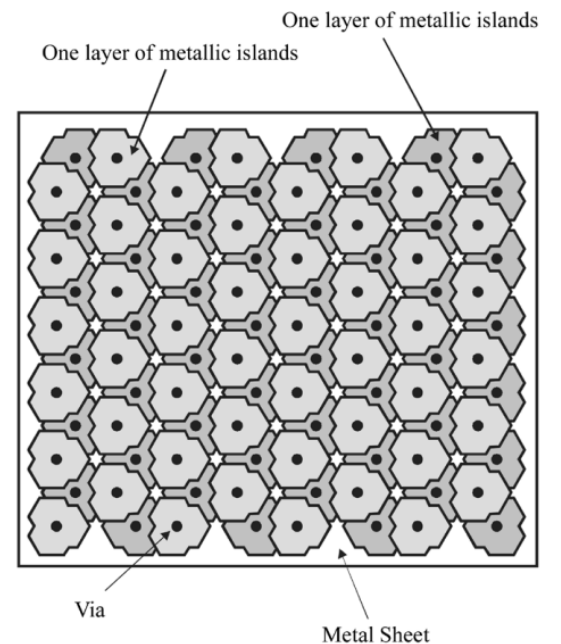

(a)

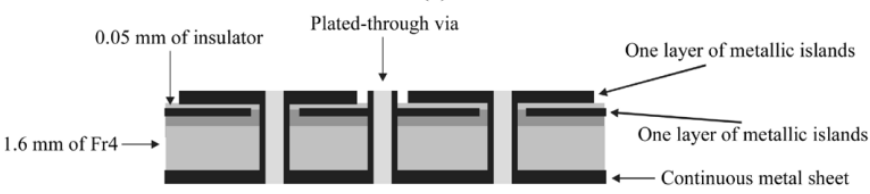

(b)

Fig. 1. (a) Top view of the high-impedance ground plane. (b) Side view of the high-impedance ground plane.

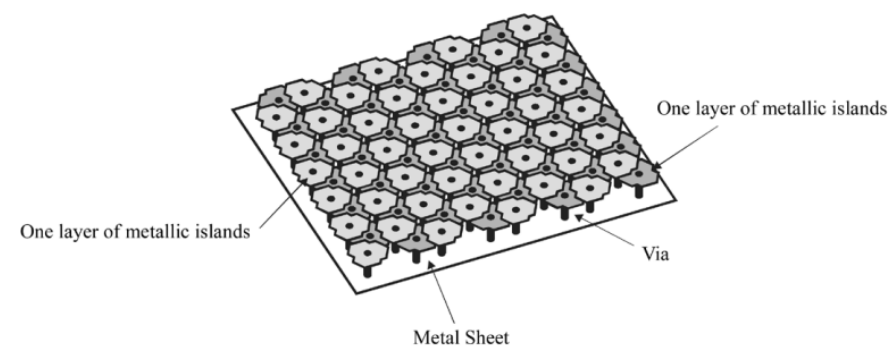

(a)

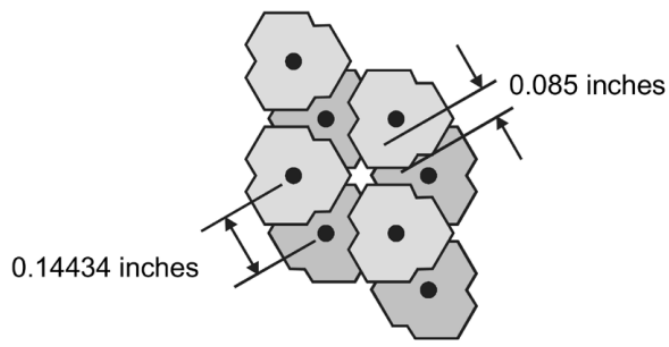

(b)

Fig. 2. (a) Side angled view of the high-impedance ground plane. (b) Dimensional detail of the high-impedance ground plane.

plane used in a cellphone handset geometry that was used in this phased array application [6]. The design process, measurement techniques, and confirmation of the bandgap for this high- 


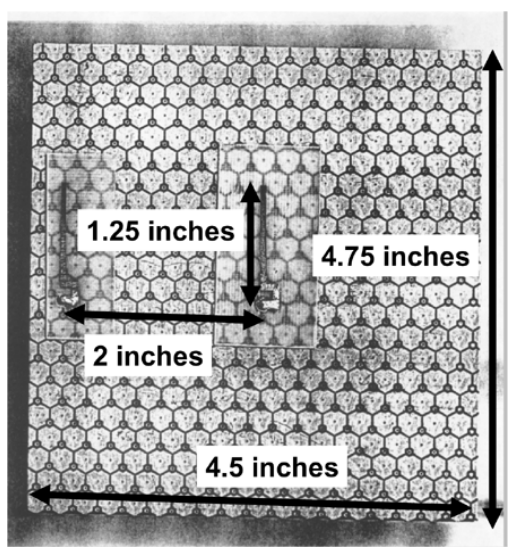

Fig. 3. Photograph of the high-impedance ground plane with dimensions. Two radiators in parallel and separated by 2 in are shown mounted on the high-impedance ground plane as in the experiment.

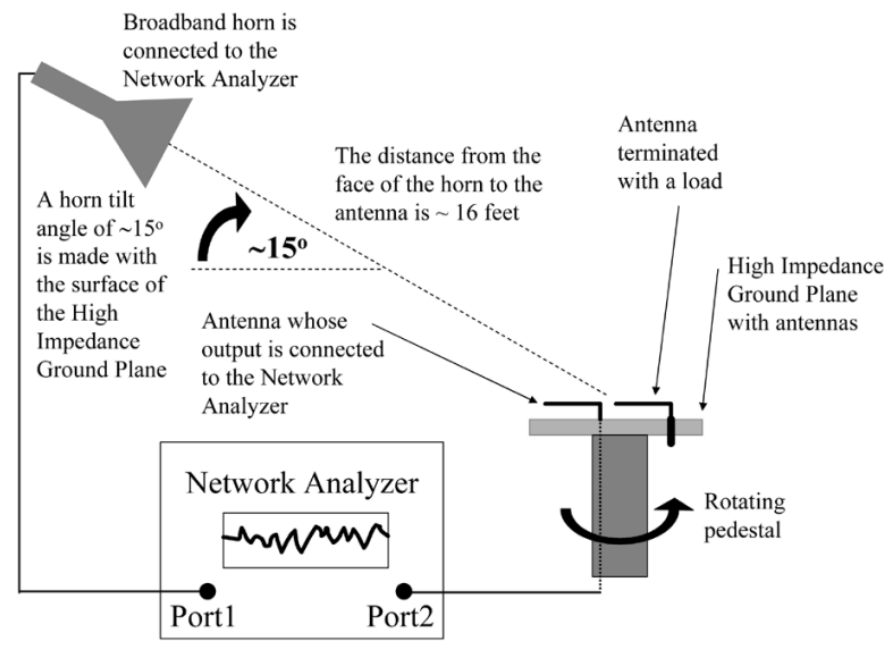

Fig. 4. Experimental setup similar to the GPS configuration to study the suppression of surface waves by the high-impedance ground plane.

impedance ground plane have already been published [5], [6]. Furthermore, general design procedures for this type of highimpedance ground planes were discussed in detail [7].

\section{EXPERIMENT, RESULTS, AND ANALYSIS}

To study the suppression of surface waves by the high-impedance ground plane, the basic setup shown in Fig. 4 was used. The height of the rotating pedestal was lowered and the horn was tilted so that there is a $15^{\circ}$ angle between the plane of the high-impedance ground plane and the pointing direction of the horn. Physical constraints limited the tilt angle to $15^{\circ}$. The setup tries to mimic a satellite-Global Positioning System (GPS) system where the horn would simulate the satellite, and the array would be a phased array GPS receiver. The actual purpose would be to steer nulls in the direction of jamming signals, so the horn may actually be simulating the jamming signal.

The experimental protocol was the following: three antennas were built using the high-impedance ground plane. All the radiating elements were identical and had the dimensions shown in

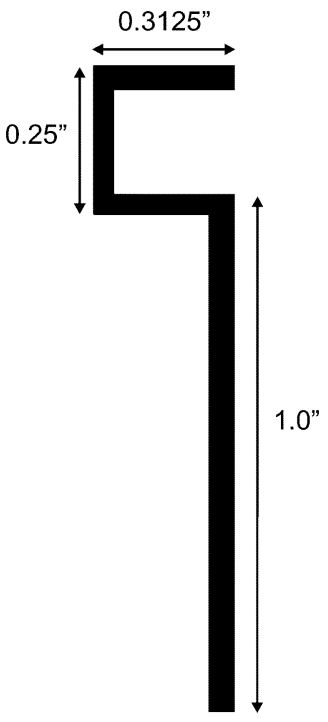

Fig. 5. Radiating element mounted on the high-impedance ground plane.

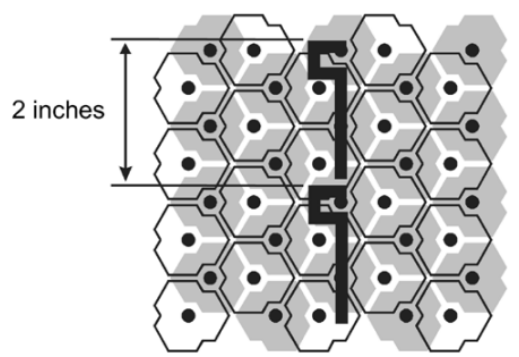

(a)

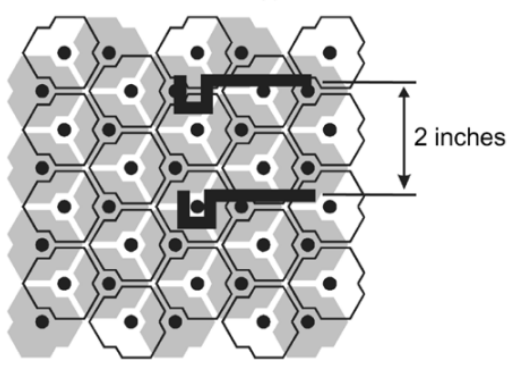

(b)

Fig. 6. Configurations in which two radiators are colinear (a), or parallel (b), to one another on a high-impedance ground plane.

Fig. 5. This radiating element was specifically matched to have good return loss for frequencies inside and outside the bandgap. The first antenna had only a single radiator, and the other two antennas had a pair of radiating elements as positioned either parallel or colinear to each other, as shown in Fig. 6. The separation of the radiators both in the parallel and colinear cases was $2 \mathrm{in}$. At $2.45 \mathrm{GHz}$, the wavelength in free space is $4.82 \mathrm{in}$, and so the separation of 2 in is well below the $\lambda / 2$ mark.

The antenna with a single radiator was rotated in an anechoic chamber, and the phase of the received signal was measured at various angles. Each antenna having two radiators was then measured in a similar manner, and the phase of the received signal was compared to that of the single radiator. This yields the effect of a second radiator on the received phase of the first, and provides a quantitative measurement of the interaction between them. The second radiator was terminated with a load. 

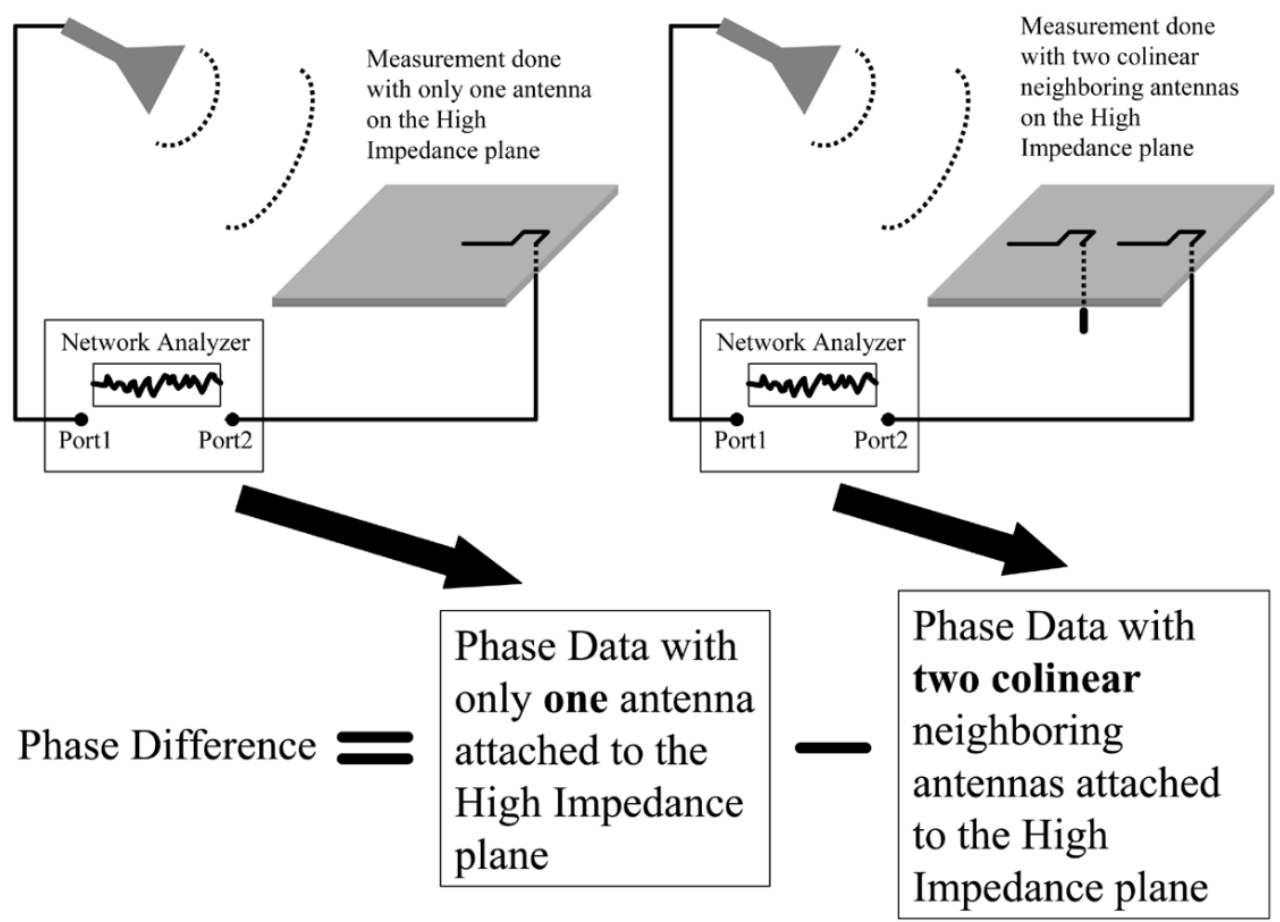

Fig. 7. Measurement procedure to analyze the effects of attaching a colinear neighboring radiator on a high-impedance ground plane. A similar test was performed on the parallel configuration of Fig. 6(b).

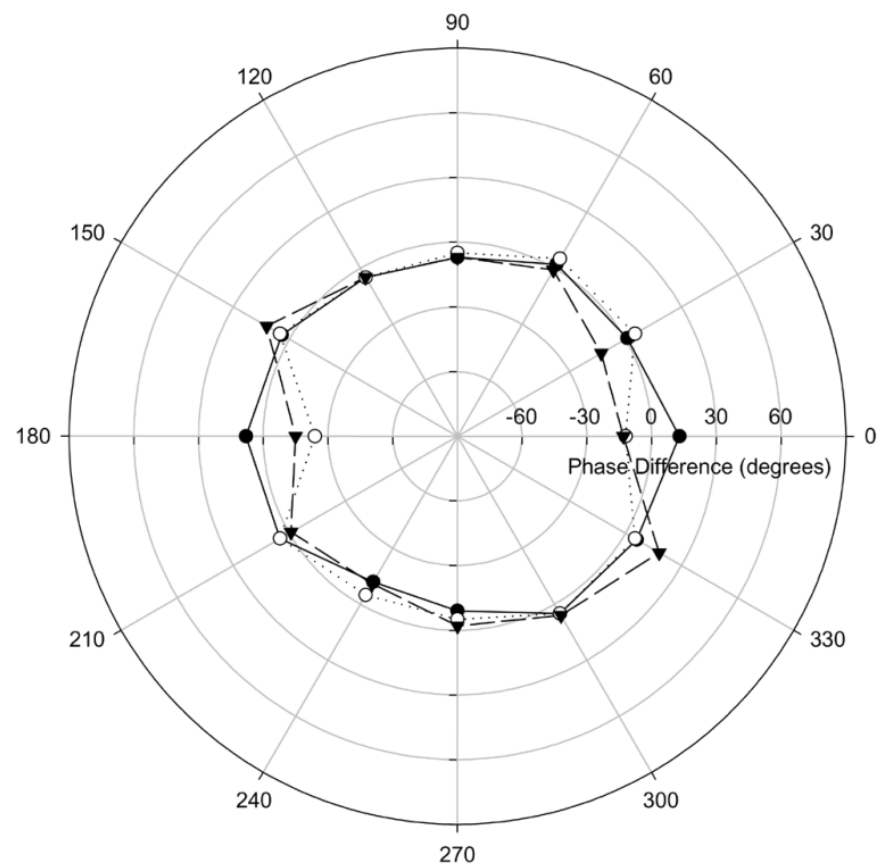

$-2.4 \mathrm{GHz}, \mathrm{SD}=7.30$
$-\mathrm{O} 2.45 \mathrm{GHz}, \mathrm{SD}=8.99$
$-\boldsymbol{\nabla} 2.5 \mathrm{GHz}, \mathrm{SD}=10.28$

Fig. 8. High-impedance ground plane with colinear radiators. The frequencies plotted are inside the bandgap.

Specifically, what was done were the following: first, a phase measurement was made with only one radiator present on the high-impedance ground plane. This data was stored on the memory (Memory 1) of the network analyzer. A second phase

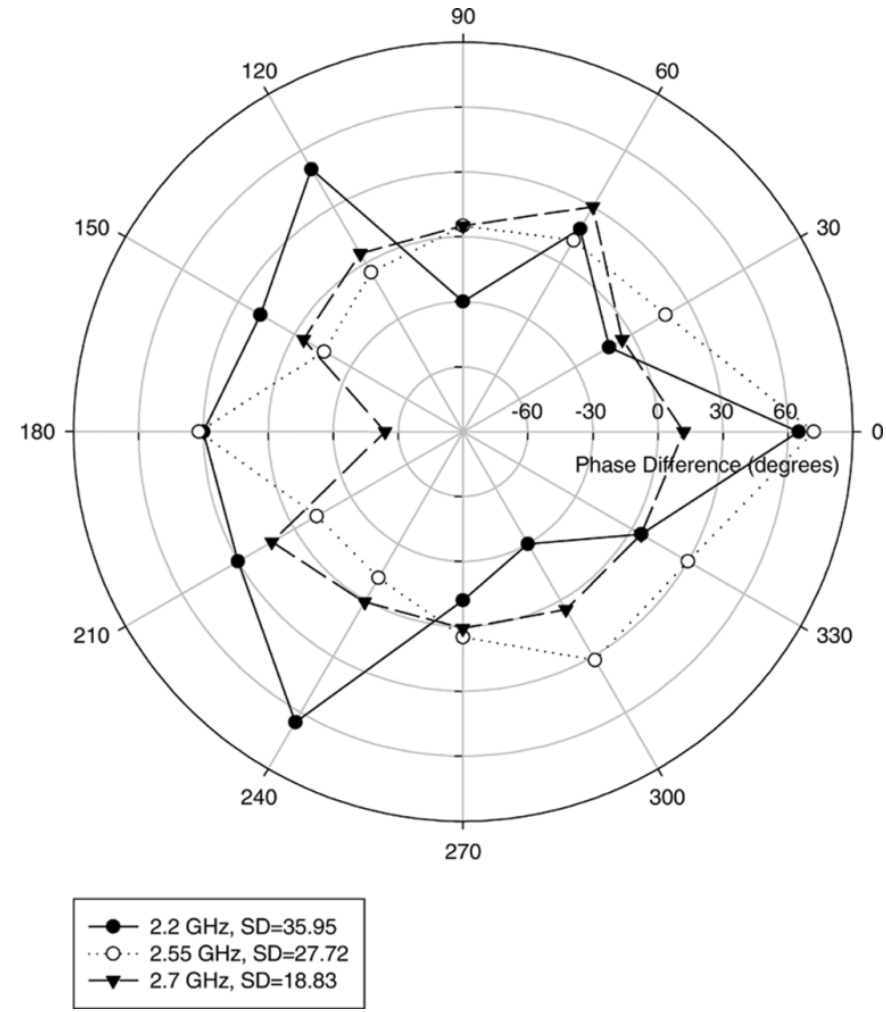

Fig. 9. High-impedance ground plane with colinear radiators. The frequencies plotted are outside the bandgap.

measurement was then taken, but this time two radiators are present on the high-impedance ground plane instead of one. This data was stored on Memory 2 of the network analyzer. Finally, Memory 2 was subtracted from Memory 1 to see how 
TABLE I

Summary of the STANDARD DeVIATIONS OF THE PHASE DiFFERENCE (IN DEGREES) AS A FUNCTION OF FREQUENCY (IN GHZ)

\begin{tabular}{|c|c|c|c|}
\hline & Frequency $(\mathrm{GHz})$ & Colinear & Parallel \\
\hline \multirow{3}{*}{ 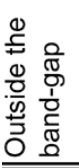 } & 2.20 & 35.95 & 33.35 \\
\hline & 2.55 & 27.72 & 41.33 \\
\hline & 2.70 & 18.83 & 61.44 \\
\hline \multirow{3}{*}{ 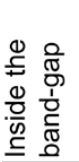 } & 2.40 & 7.30 & 23.28 \\
\hline & 2.45 & 8.99 & 26.17 \\
\hline & 2.50 & 10.28 & 20.57 \\
\hline
\end{tabular}
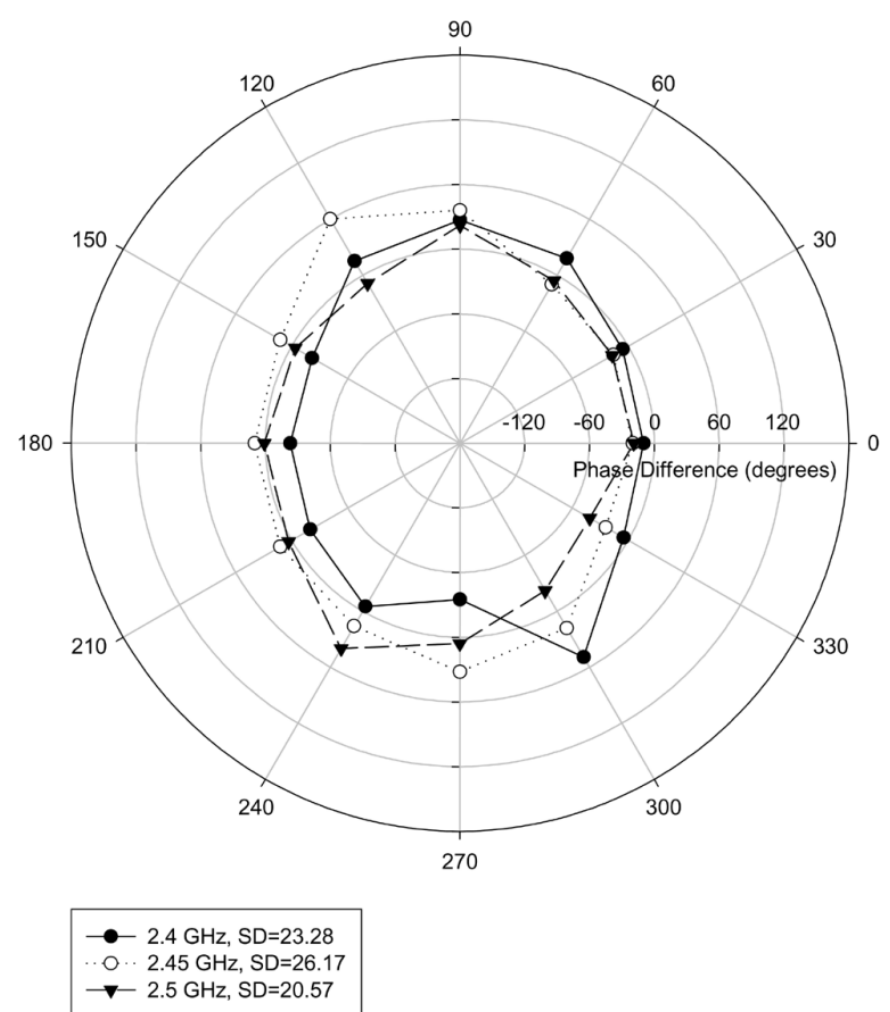

Fig. 10. High-impedance ground plane with radiators that are parallel to one another. The frequencies plotted are inside the bandgap.

the phase measurement was affected by the presence of the second radiator. This protocol was done for $30^{\circ}$ rotational angle increments, and for frequencies inside and outside the bandgap. The measurement procedure is shown in Fig. 7 for the colinear radiators layout, and the same procedure was done for the parallel radiators layout.

The three polar plots shown in Fig. 8 are for three frequencies within the surface wave bandgap: $2.4,2.45$, and $2.5 \mathrm{GHz}$. In this particular case, the two radiators are colinear. Fig. 9 shows the same radiators measured outside the band gap, at frequencies of 2.2, 2.55, and $2.7 \mathrm{GHz}$. For frequencies in the bandgap, there is very little phase variation for all rotation angles. For frequencies outside of the bandgap, the deviation from zero phase difference is more pronounced. This is because in the bandgap, the suppression of surface currents minimizes the inter-element

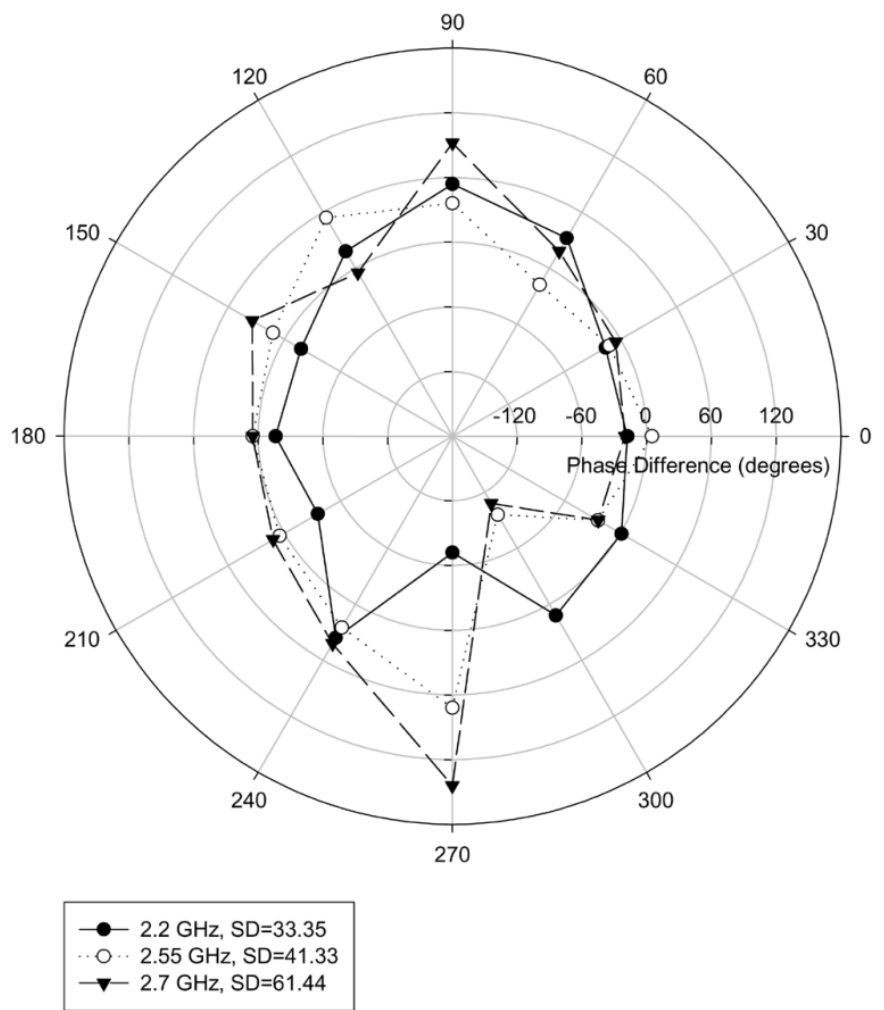

Fig. 11. High-impedance ground plane with radiators that are parallel to one another. The frequencies plotted are outside the bandgap.

coupling between the two radiators. To quantify this, the standard deviation from zero phase difference was calculated and summarized in Table I.

The same measurements were also performed using the pair of parallel, noncolinear radiators, and the results are plotted in Figs. 10 and 11.

The phase difference for the parallel radiators layout [Fig. 6(b)] is greater than the phase difference for the colinear radiators layout [Fig. 6(a)]. This is observed to be true for all frequencies, both inside and outside of the bandgap. Intuitively, this makes sense, since in the parallel radiators layout, the two radiators are more likely to interfere than the straight, colinear radiators layout. This happens for two reasons: the surface supports leaky TE waves that can still couple the radiators in the parallel case, and the radiators produce a weaker electric field in the direction of the colinear case.

Nevertheless, the same trends can be observed in the parallel radiators layout as in the straight, colinear radiators layout. Namely, frequencies lying inside the bandgap have less phase difference than frequencies outside of the bandgap. The calculation of the standard deviations once again quantifies this phase difference as a function of frequency.

The results of this paper are important for phased array applications. In trying to null out a jammer, the proper weighting function for combining all of the antenna elements must be accurately determined. If the elements interact, this will affect the weighting function, thereby requiring complicated characterization of the antenna for all null angles. The design should be simplified by the suppression of this interaction as accomplished by the high-impedance ground plane. 


\section{SUMMARY}

A high impedance ground plane (three-layer hexagonal structure) was analyzed in the microwave frequency regime of 2.2 to $2.8 \mathrm{GHz}$. The bandgap of the three-layer hexagonal structure was designed to be from 2.35 to $2.55 \mathrm{GHz}$, and this bandgap was confirmed experimentally.

The ability of the high impedance ground plane to suppress surface waves was used in a phased array application. In the bandgap, there is a suppression of surface waves so that interelement radiator coupling is minimized. Outside of the bandgap, the coupling between radiator elements is greater so that one radiator greatly influences its neighboring radiators.

\section{REFERENCES}

[1] D. Sievenpiper, L. Zhang, R. F. J. Broas, N. Alexopolous, and E. Yablonovitch, "High-impedance electromagnetic surfaces with a forbidden frequency band," IEEE Trans. Microwave Theory Tech., vol. 47, no. 11, pp. 2059-2074, Nov. 1999.

[2] D. Sievenpiper, "High-Impedance electromagnetic surfaces," Ph.D. dissertation, Dept. Elect. Eng., Univ. California at Los Angeles, Los Angeles, CA, 1999.

[3] D. Sievenpiper and E. Yablonovitch, "Circuit and method for eliminating surface currents on metals," U.S. Patent $6262495,1999$.

[4] J. J. Lee, R. F. J. Broas, S. Livingston, and D. Sievenpiper, "Flushmounted antennas on Hi-Z ground planes," in Proc. IEEE Antennas and Propagation Soc. Int. Symp., vol. 3, Jun. 2002, pp. 764-767.

[5] R. F. J. Broas, "Experimental characterization of high impedance electromagnetic surfaces in the microwave frequency regime," M.S. thesis, Dept. Elect. Eng., Univ. California at Los Angeles, Los Angeles, CA, 1999.

[6] R. F. J. Broas, D. F. Sievenpiper, and E. Yablonovitch, "A high-impedance ground plane applied to a cellphone handset geometry," IEEE Trans. Microwave Theory Tech., vol. 49, no. 7, pp. 1262-1265, Jul. 2001.

[7] S. Clavijo, R. E. Diaz, and W. E. McKinzie III, "Design methodology for Sievenpiper high-impedance surfaces: An artificial magnetic conductor for positive gain electrically small antennas," IEEE Trans. Antennas Propag., vol. 51, no. 10, pp. 2678-2690, Oct. 2003.

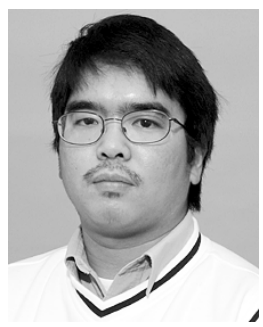

Romulo F. Jimenez Broas was born in Cavite, Philippines. He received the A.B. degree in physics from the University of California at Berkeley, in 1996 and the M.Sc. degree in electrical engineering from the University of California at Los Angeles, in 1999.

He was a Member of Technical Staff at TRW from 1997 to 1998, and is currently a Senior Electrical Engineer at Raytheon, El Segundo, CA, since 1999. He published Math Problems Everyone Should Know How to Solve, and Their Solutions (Carson, CA: Manel Publications, 2004) and has 14 publications, including one U.S. patent and one pending patent. His research interests include solid state physics, electromagnetics and antennas, mathematics, and biophysics.

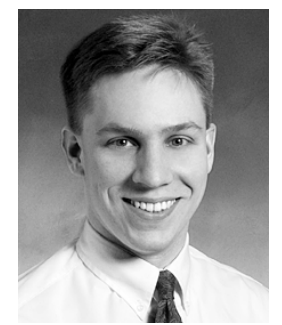

Daniel F. Sievenpiper (S'95-M'98-SM'04) received the B.S. and Ph.D. degrees in electrical engineering from the University of California at Los Angeles, in 1994 and 1999, respectively.

$\mathrm{He}$ is currently a Senior Research Staff Scientist with HRL Laboratories, Malibu, CA, where he works in the field of electromagnetics, antennas, and wireless communication. His interests include novel electromagnetic materials, low-cost beam steering, RF MEMS for advanced antennas and radio front ends, and new communication system architectures. He has more than 20 U.S. patents and more than 40 refereed journal publications and conference papers.

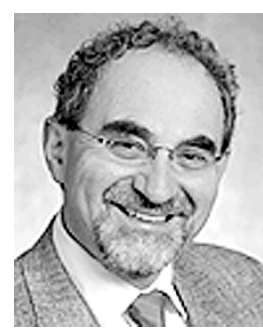

Eli Yablonovitch (M'75-SM'90-F'92) received the Ph.D. degree in applied physics from Harvard University, Cambridge, MA, in 1972.

He worked for two years at Bell Telephone Laboratories, and then became a Professor of applied physics at Harvard. In 1979, he joined Exxon to do research on photovoltaic solar energy and in 1984, he joined Bell Communications Research, where he was a Distinguished Member of Staff, and also the Director of Solid-State Physics Research. In 1992, he joined the University of California at Los Angeles, where he is Professor of electrical engineering. His work has covered a broad variety of topics: nonlinear optics, laser-plasma interaction, infrared laser chemistry, photovoltaic energy conversion, strained-quantum-well lasers, and chemical modification of semiconductor surfaces. Currently his main interests are in optoelectronics, high speed optical communications, high efficiency light-emitting diodes and nanocavity lasers, photonic crystals at optical and microwave frequencies, quantum computing, and quantum communication.

Prof. Yablonovitch is a Fellow of the Optical Society of America and the American Physical Society, a Life Member of Eta Kappa Nu, and a Member of the National Academy of Engineering and the National Academy of Sciences. He has been awarded the Adolf Lomb Medal, the W. Streifer Scientific Achievement Award, the R.W. Wood Prize, the Julius Springer Prize, and numerous additional awards. He was a Founder of the W/PECS series of Photonic Crystal International Workshops that began in 1999. (PECS VI will be held in Crete in June 2005.) 